\title{
ON THE ORDER OF THE FINITE TEMPERATURE DECONFINEMENT PHASE TRANSITION IN Z(2) AND Z(3) LATTICE GAUGE THEORIES
}

\author{
R.V. GAVAI \\ Zentrum für interdisziplinäre Forschung, Bielefeld, Fed. Rep. Germany \\ and Fakultät für Physik der Universität Bielefeld, Bielefeld, Fed. Rep. Germany \\ and \\ F. KARSCH \\ CERN, Geneva, Switzerland
}

Received 4 March 1983

\begin{abstract}
Using Monte Carlo methods, we show that the finite temperature deconfinement phase transition in $Z(3)$ lattice gauge theory is of first order by presenting evidence for the coexistence of the two phases in the transition region. A similar study for the $Z(2)$ theory, however, failed to yield any such evidence, thus indicating the corresponding transition to be of higher order.
\end{abstract}

Recent investigations of finite temperature gauge theories using Monte Carlo methods on lattices, have yielded interesting results. The existence of a deconfining phase transition for SU(2) and SU(3) lattice gauge theories, known previously at strong coupling [1], has been shown to exist also for intermediate values of couplings $(2,3)$, which allowed an extraction of physical values for the transition temperature of the corresponding continuum theories using renormalization group arguments. It is, of course, desirable to understand more about the nature of these deconfining phase transitions and the most natural question one would like to ask to this end is about the order of the phase transition. Predictions about the order of the deconfinement phase transition in a host of pure lattice gauge theories have been made by Svetitsky and Yaffe [4]. They argue that due to the fact that the deconfinement phase transition can be characterized by the spontaneous breakdown of a global symmetry, i.e., the $\mathrm{Z}(N)$ centre symmetry for $\operatorname{SU}(N)$ groups, the critical behaviour of $\mathrm{SU}(N)$ and $\mathrm{Z}(N)$ theories should be similar provided that the corresponding transitions are continuous. Relating the $(d+1)$-dimensional gauge models at finite temperature to a $d$-dimen- sional spin model with the same global symmetry, they predict the order of the phase transition in models with a global Z(3) symmetry to be first order, whereas in models with $Z(2)$ symmetry, the transition may be of second order. On the basis of Monte Carlo studies it has been recently claimed that the deconfinement phase transitions in SU(2) and SU(3) are indeed second and first order, respectively [5], basically using the sharpness of the SU(3) transition as a criterion for its first order nature. However, we would like to emphasize that Monte Carlo simulations of lattice gauge theories allow a clear distinction between discontinuous and continuous phase transitions. The coexistence of two phases at temperatures around the critical temperature $T_{\mathrm{c}}$, characteristic for a discontinuous phase transition can be shown in Monte Carlo runs at fixed temperature using different start configurations. This has been observed by Creutz et al. in their study of $Z(N)$ gauge theories at zero temperature [6].

The aim of our work is to see to what extent the order of finite temperature phase transitions can be determined through a search for coexisting phases. We have studied $Z(2)$ and $Z(3)$ lattice gauge theories in three space dimensions for this purpose. The critical 
behaviour of these models is expected to be related to that of a 3-d Ising model and a 3-d three-state Potts model, which yield second and first order transitions, respectively [7]. Our analysis may, therefore, serve as a check of some of the basic predictions of ref. [4]. Alternatively, combined with results such as those of ref. [5], our analysis may test the relevance of the underlying global symmetry group for the order of the deconfinement transition.

The thermodynamics of $Z(N)$ gauge theories in three space dimensions can be obtained from the partition function

$Z=\operatorname{tr} \exp (-\beta H)$,

where the trace is to be taken over physical states [ 8 , 9] only, $\beta=1 / T$ is the inverse temperature and the hamiltonian $H$ is given by [8]

$$
\begin{aligned}
H= & -\sum_{\text {plaquettes }} \hat{C}_{n, \mu} \hat{C}_{n+\mu, \nu} \hat{C}_{n+\nu, \mu}^{+} \hat{C}_{n, \nu}^{+} \\
& \cdot \gamma \sum_{\text {links }} \hat{R}_{n, \mu}+\text { h.c } .
\end{aligned}
$$

Here the plaquettes and links are defined in a threedimensional cubic lattice and the operators $\hat{R}_{n, \mu}$ and $\hat{C}_{n, \mu}$, which are associated with a link joining neighbouring points $n, n+\mu$ satisfy the following conditions

$\hat{R}_{n, \mu} \hat{C}_{n, \mu}=\exp (-\mathrm{i} 2 \pi / N) \hat{C}_{n, \mu} \hat{R}_{n, \mu}$,

$\hat{R}_{n, \mu}^{N}=\hat{C}_{n, \mu}^{N}=1$.

The above Hamiltonian is self-dual for all $N$, the self-dual point being $\gamma=1$. Further, for $N=2$ and 3 , the cases of our interest, it is identical to the hamiltonian of the $N$ state Potts model whose finite temperature behaviour has been discussed by Goldschmidt and Shigemitsu [9], using large $N$ expansion. Fig. 1

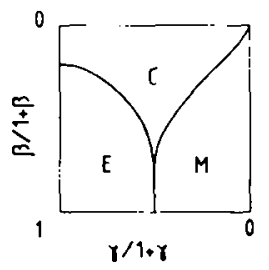

Fig. 1. The chematic phase diagram for $Z(2)$ and $Z(3)$ lattice gauge theories at finite temperature in three space dimensions. The notation is explained in the text. shows their generic phase diagram for both $Z(2)$ and $Z(3)$, redrawn in our notation. These models have three different phases in which either only "electric" charges [9] or only "magnetic" charges or nothing is confined, labelled in fig. 1 as E, M, C, respectively. At zero temperature, self-duality predicts a phase transition at $\gamma_{c}=1$, separating the phases $E$ and $M$. At finite temperatures and $\gamma>1$, however, there is a deconfinement phase transition which separates the phases $\mathrm{E}$ and $\mathrm{C}$. Due to the similarity of this phase transition with what one has in $\mathrm{SU}(N)$ gauge theories, we will be interested in the following in the deconfinement transition of electric charges.

The problem of the evaluation of the partition function, eq. (1), can be reformulated in to that of the calculation of the partition function of a $Z(N)$ gauge theory defined on a $(3+1)$-dimensional euclidean hypercubic lattice, using standard techniques $[10,11]$. For $N=2,3$ the partition function takes on a particularly simple form

$$
\begin{aligned}
Z= & \lim _{N_{\beta} \rightarrow \infty} \sum_{\left\{U_{n, \mu}\right\}} \exp \left(-a_{\beta} \sum_{\left\{\mathrm{p}_{\mathrm{s}}\right\}}(1-\operatorname{Re} U U U U)\right. \\
& \left.-g\left(a_{\beta, \gamma}\right) \sum_{\left\{\mathrm{p}_{\beta}\right\}}(1-\operatorname{Re} U U U U)\right),
\end{aligned}
$$

where $a_{\beta}=2 \beta / N_{\beta}$ and the coupling $g\left(a_{\beta}, \gamma\right)$ is given by

$$
\begin{aligned}
& g\left(a_{\beta}, \gamma\right)=\frac{N-1}{N} \ln \left(\frac{\exp \left[N a_{\beta} \gamma /(N-1)\right]+N-1}{\exp \left[N a_{\beta} \gamma /(N-1)\right]-1}\right), \\
& N=2,3 .
\end{aligned}
$$

In eq. (4) $\left\{p_{s}\right\},\left\{p_{\beta}\right\}$ denote the set of all plaquettes containing respectively zero or two links in the newly added inverse temperature direction and $\left\{U_{n, \mu}\right\}$ is the set of all link variables in the $(3+1)$-dimensional lattice, taking on the values

$U_{n, \mu}=\exp (\mathrm{i} 2 \pi k / N), \quad k=0,1, \ldots, N-1$.

The trace operation in eq. (1) restricts the $U_{s}$ in the $\beta$ direction as below:

$U_{(n, 1), 0}=U_{\left(n, N_{\beta}\right), 0}$.

The confinement region of electric charges is characterized by an order parameter which is zero in this regime and non-zero otherwise. For this purpose one uses the expectation value of the thermal Wilson loop 
$\langle L(n)\rangle=\left\langle\prod_{k=1}^{N_{\beta}} U_{(n, k), 0}\right\rangle$,

where $\langle\ldots$.$\rangle denotes thermal averaging: \langle X\rangle=Z^{-1} \operatorname{tr} X$ $X \exp (-\beta H)$. As $L(n)$ transforms non-trivialiy under global $Z(N)$ transformations, a non-vanishing expectation value signals a spontaneous breakdown of this global symmetry.

Using a $8^{3} \times 4$ lattice, we have studied the above order parameter in the vicinity of the deconfinement phase transition for both $Z(2)$ and $Z(3)$ lat tice gauge theorics. At zero temperature $(\beta=\infty)$ this transition occurs at the self-dual point $\gamma_{\mathrm{c}}=1$ and has been shown to be first order [6]. For finite but low temperatures the phase boundary is still at $\gamma_{c}=1$, as calculated in ref. [8], and the phase transition from the electric into the magnetic confinement phase is still expected to be of first order. At sufficiently high temperature, however, we expect $\gamma_{c}>1$ and moreover, the $Z(2)$ transition is supposed to be no longer of first order but of second order, whereas the one in $\mathrm{Z}(3)$ is still expected to be of first order [4]. Therefore, we should always be able to find a region of couplings and temperatures where one observes the coexistence of the deconfined phase $(\langle L\rangle>0)$ and the confined phase $(\langle L\rangle \sim 0)$ indicating a first order phase transition, except for the case when $\gamma_{c}>1$ for $Z(2)$.

In order to verify first whether for large enough $\beta$ one still has a first order transition at $\gamma=1$, we fixed $\gamma=1$ and chose $\beta=0.75$ for both $Z(2)$ and $Z(3)$. Using a standard heat bath algorithm [12], we let the lattice evolve from ordered and random start configurations. Fig. $2 \mathrm{a}$ and $2 \mathrm{~b}$ show the results of this calculation for the order parameter $\langle|L|\rangle$ for $Z(2)$ and $\langle\operatorname{Re}$ $L^{3}$ ) for $Z(3)^{\dagger 1}$. One clearly sees that the system equilibrates quickly and exhibits a distinct two-phase behaviour. We thus confirm the results of ref. [9] about the position of the transition and also the naive expectation that the transition continues to be first order [4]. We progressively decreased $\beta$ in both the cases and found similar results as in fig. 2 for a substantial range

${ }^{*}$ Because of the exact $Z(N)$ symmetry of the problem $(|L|)$ for $Z(2)$ and $\left(\operatorname{Re} L^{3}\right\rangle$ for $Z(3)$ is a convenient quantity to consider in Monte Carlo simulations on finite lattices $[2,3]$. Here $L$ denotes an average of $L(n)$ over the lattice after each iteration.
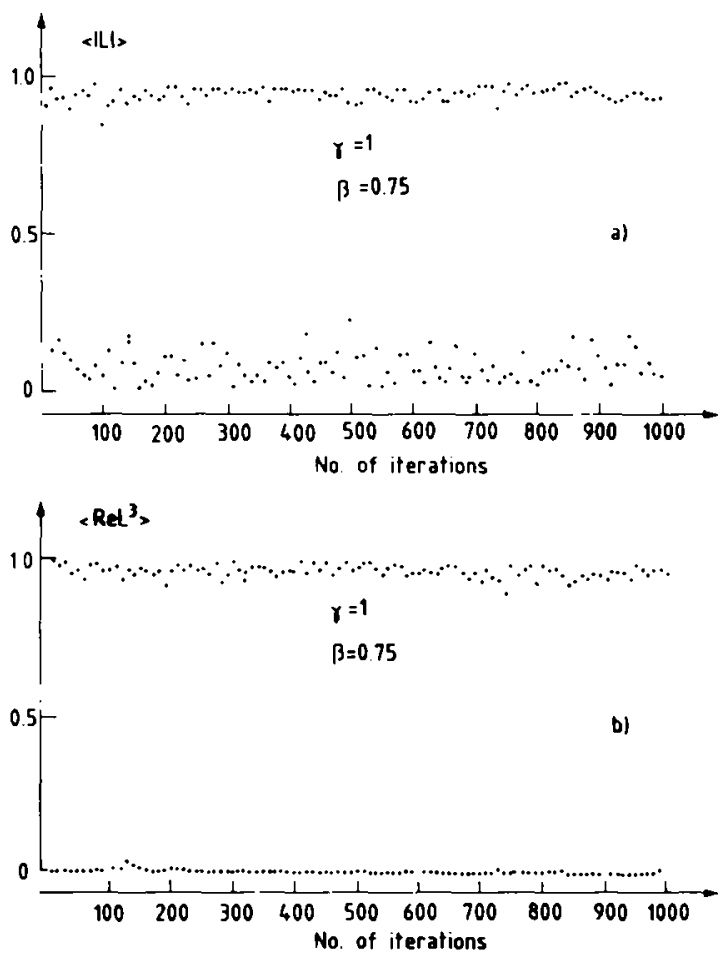

Fig. 2. The order parameter for a random and ordered start at $\gamma=1$ and low temperature $\beta=0.75$, i.e., (a) $\langle|L|\rangle$ for $Z(2)$; and (b) $\left\langle\operatorname{Re} L^{3}\right\rangle$ for $Z(3)$.

of $\beta$, although the fluctuations in the random (confinement) phase seem to become increasingly bigger as we lowered $\beta$ still further. At $\beta=0.5$ in the case of $Z(2)$ and $\beta=0.65$ in the case of $Z(3)$, we found that the coexistence of the phases was no more to be seen at $\gamma=1$; instead both the ordered and random start soon approached each other and $\langle|L|\rangle,\left\langle\operatorname{Re} L^{3}\right\rangle$ was distinctly bigger than zero, as we show in figs. $3 a$ and $4 a$, respectively. Clearly, for these values of $\beta$ and $\gamma$ in the respective cases, one is in the deconfined phase (phase $\mathrm{C}$ in fig. 1) and the deconfining transition at these $\beta$ values was no more at $\gamma=1$. To determine the $\gamma$ at which the transition takes place, we fixed the $\beta$ value in each case and increased now $\gamma$. In both the $Z(2)$ and $Z(3)$ cases, we found that at $\gamma=1.05$ the system, when allowed to equilibrate from an ordered and random start, approached towards the random phase, with $\langle|L|\rangle,\left\langle\operatorname{Re} L^{3}\right\rangle$ vanishingly small in each case, as shown in figs. $3 \mathrm{e}$ and $4 \mathrm{~d}$, respectively. Thus $\gamma=1.05$ at $\beta=0.5[\beta=0.65]$ clearly corresponds already to the confining phase for the $Z(2)[Z(3)]$ theory. 


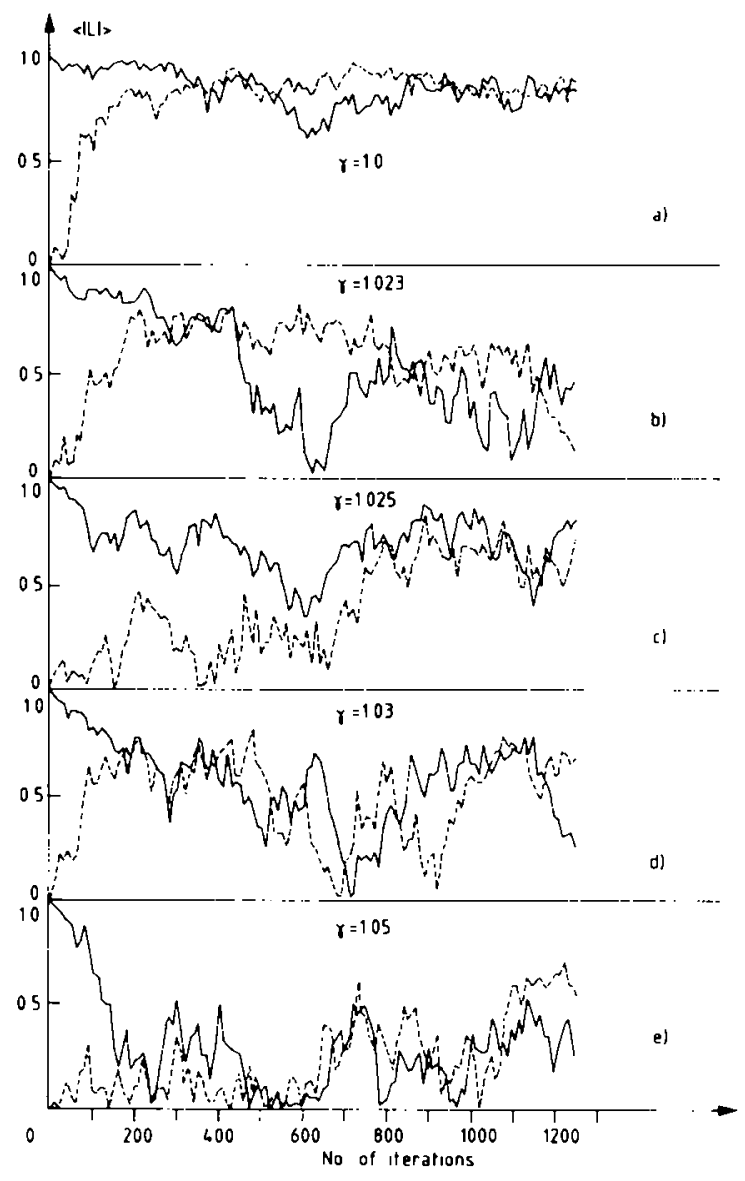

Fig. 3. The evolution of the order parameter $\langle|L|\rangle$ for the $Z(2)$ theory from random (dashed line) and ordered (full line) start configurations at $\beta=0.5$ and (a) $\gamma=1.0$; (b) $\gamma=1.023$; (c) $\gamma=1.025$; (d) $\gamma=1.03$; and (e) $\gamma=1.05$.

In order to find out the phase transition point in each case, and check whether the transition is still of first order on the boundary of the phases $\mathrm{E}$ and $\mathrm{C}$ (fig. 1), we fixed $\beta$ at the respective value used above and then varied $\gamma$ in very small steps in the range 1.0 $<\gamma<10.5$. At each pair of $\beta, \gamma$ values, the behaviour of the system evolving from an ordered and a random start was compared. We found a narrow region of $\gamma$ values $(\Delta \gamma \sim 0.0025)$ for the case of the $Z(3)$ theory where one observes coexisting phases, as we show in figs. $4 \mathrm{~b}$ and $4 \mathrm{c}$ for $\gamma=1.03$ and 1.0325 , respectively. For the case of $Z(2)$, however, we found no such behaviour for any $\gamma$ value. As we show in the three typical runs near the transition point in figs. $3 \mathrm{~b}, 3 \mathrm{c}$ and

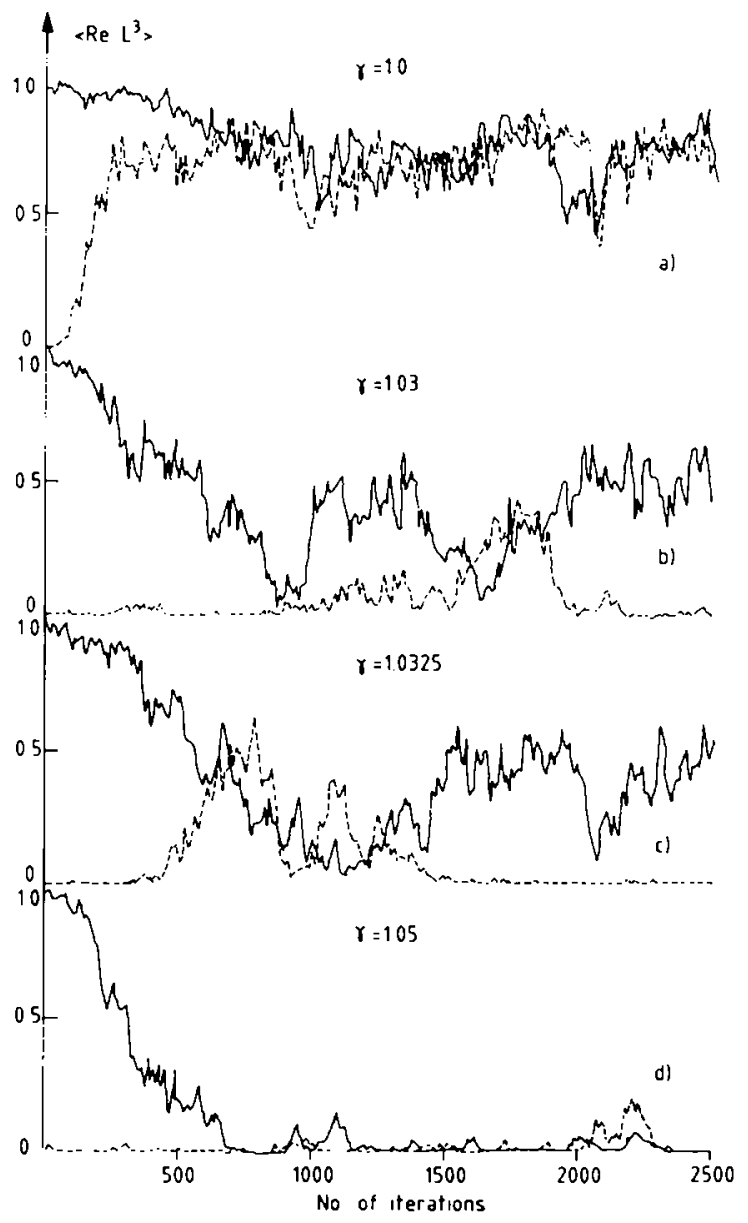

Fig. 4. Same as fig. 3, but for $\left\langle\operatorname{Re} L^{3}\right\rangle$ of the $Z(3)$ theory at $\beta$ $=0.65$ and (a) $\gamma=1.0 ;$ (b) $\gamma=1.03$, (c) $\gamma=1.0325$; and (d) $\gamma$ $=1.05$.

$3 \mathrm{~d}$ there were always very large oscillations in both the phases, but even if one averaged over the oscillatory behaviour, the mean $\langle|L|\rangle$ from the two start configurations approached each other, showing thus an absence of any coexistence of two phases $¥ 2$.

The large fluctuations which one observes in the order parameter, as in figs. $3 b-3 d$, further indicate that the transition in the case of $Z(2)$ is perhaps of higher order. It is, of course, not possible from these

\footnotetext{
$\neq 2$ We have performed the same exercise at many more $\gamma$ values in the range $1<\gamma<1.05$. All the figures look similar to figs. 3b-. 3d; only the oscillations get smaller, as one moves away from $\gamma \sim 1.025$.
} 
data to determine whether it is indeed of second order, as claimed in ref. [4], although one can, in principle, check their prediction by calculating quantities such as the specific heat; a diverging peak (on finite lattices, one can see an increase in its height with increasing size of the lattice) at the transition point should be observed if the transition is of second order.

We have shown the deconfinement phase transition in $Z(2)$ and $Z(3)$ lattice gauge theories, i.e., the transition between the phases $E$ and $C$ of fig. 1 , to be continuous and of first order, respectively. As pointed out earlier, the implications of these results for SU(2) and SU(3) are quite interesting and it would thus be nice to see whether such coexistence of phases can also be found for the SU(3) theory which is also predicted to have a first order transition on the basis of exactly the same arguments which predict the $Z(3)$ transition to be of first order [4]. Though so far no evidence has been presented in the literature for coexisting phases in the case of SU(3) to support the claim that its transition is of first order, we understand that work is in progress and will be reported soon $[5,13]$.

We thank T. Celik, P. Hasenfratz and H. Satz for discussions.

\section{References}

[1] A.M. Polyakov, Phys. Lett. 72B (1978) 477; L. Susskind, Phys. Rev. D20 (1979) 2610.

[2] L. McLerran and B. Svetitsky, Phys. Lett. $98 \mathrm{~B}$ (1981) 195

J. Kuti, J. Polonyi and K. Szlanchanyi, Phys. Lett. 98B (1981) 199;

J. Engels, F. Karsch, H. Satz and I. Montvay, Phys. Lett. 101B (1981) 89

[3] K. Kajantie, C. Montonen and E. Pietarinen, Z. Phys. C9 (1981) 253.

[4] B. Svetitsky and L.G. Yaffe, Nucl. Phys. B210 [FS6] (1982) 423 .

[5] J. Kogut, et al., Phys. Rev. Lett. 50 (1983) 393.

[6] M. Creutz, L. Jacobs and C. Rebbi, Phys. Rev, Lett. 42 (1979) 1390; Phys. Rev. D20 (1979) 1915.

[7] J.S. Knak Jensen and O.G. Mouritsen, Phys. Rev. Lett. 43 (1979) 1736.

[8] J. Kogut, R.B. Pearson, J. Shigemitsu and D.K. Sinclair, Phys. Rev. D22 (1980) 2447.

[9] Y.Y. Goldschmidt and J. Shigemitsu, Nucl. Phys. B200 [FS4] (1982) 149.

[10] M. Suzuki, Prog. Theor. Phys. 56 (1976) 1454.

[11] J. Engels, I. Karsch and H. Satz, Nucl. Phys. B205 [FS5] (1982) 545.

[12] M. Creutz, Phys. Rev. D21 (1980) 2308.

[13] T. Celik, J. lingels and H. Satz, in preparation. 